\title{
LA PROFESIÓN ACADÉMICA EN CHILE: PERSPECTIVAS DESDE UNA ENCUESTA INTERNACIONAL
}

\author{
Andrés Bernasconi ${ }^{1}$ \\ Paulina Berríos Cortés ${ }^{2}$ \\ Pamela Guzmán ${ }^{3}$ \\ Daniela Véliz ${ }^{4}$ \\ Sergio Celis ${ }^{5}$
}

\section{RESUMEN}

Este artículo presenta los primeros resultados para Chile de la encuesta internacional de la profesión académica APIKS (Academic Profession in the Knowledge-Based Society), aplicada a los académicos de 11 universidades entre fines de 2018 y principios de 2019. Las universidades participantes incluyen los tres tipos principales de universidades en Chile: estatales, privadas con aporte estatal (conocidas también como G9) y privadas sin aporte estatal. Respondieron la encuesta, de manera completa, 1.258 académicos y académicas. Se presentan aquí, descriptivamente, los resultados de los siguientes módulos de la encuesta: distribución del tiempo de los académicos en las diversas funciones universitarias, características de la labor de docencia, características del trabajo de investigación y perfil de las actividades de vinculación con el medio. También se informan los resultados de la experiencia de los académicos en etapas formativas de sus carreras: profesores instructores y asistentes. Los principales resultados sugieren que la docencia de pregrado sigue siendo la labor central de los académicos en todos los tipos de universidades, que una cuarta parte del tiempo se dedica a la gestión, que los modos de producción de conocimiento, con altos niveles de colaboración nacional e internacional, enfatizan la publicación de artículos en revistas por sobre otras formas de comunicación científica, y que los vínculos con el medio privilegian, como socios, a otras universidades, al gobierno y a las ONG, en desmedro de la empresa privada. También aparece que los apoyos de las universidades a sus académicos, en etapas formativas, son aún incipientes.

Conceptos clave: APIKS, profesión académica, trabajo académico, académicos en etapa formativa, docencia, investigación, vinculación con el medio.

1 Pontificia Universidad Católica de Chile, Santiago, Chile. Contacto: abernasconi@uc.cl

2 Universidad de Chile, Santiago, Chile. Contacto: berrios.paulina@uchile.cl

3 Pontificia Universidad Católica de Chile, Santiago, Chile. Contacto: paguzmans@uc.cl

4 Pontificia Universidad Católica de Chile, Santiago, Chile. Contacto: dvelizc@uc.cl

5 Universidad de Chile, Santiago, Chile. Contacto: scelis@uchile.cl 


\title{
ACADEMIC PROFESSION IN CHILE: PERSPECTIVES FROM AN INTERNATIONAL SURVEY
}

\begin{abstract}
In this article, we present the first results of the Chilean version of the international survey of the academic profession APIKS (Academic Profession in the Knowledge-Based Society) applied to faculty in 11 Chilean universities between the end of 2018 and early 2019. Participating institutions include universities in the three major groupings: public universities, private universities with state support (also known as G9), and private universities without state support. 1,258 academics answered the survey in full. The paper presents descriptively the results of the following modules of the survey: distribution of time across the various university functions, characteristics of the teaching work, characteristics of research activities, and third mission activities. We also report on the faculty's experience in the early stages of their careers: instructors and assistant professors. The main results suggest that undergraduate teaching remains the major task of faculty in all kinds of universities. One-fourth of their time is dedicated to management. Types of knowledge production, with high national and international collaboration levels, emphasize publication of journal articles over other forms of scientific communication. Outreach activities are directed mostly to other universities, the government, and NGOs over the business sector. We also show that university support to early-career faculty is still incipient.
\end{abstract}

Key concepts: APIKS, academic profession, academic work, early career academics, teaching, research, outreach. 


\section{Antecedentes}

Si bien el estudio de la profesión académica contemporánea, en perspectiva internacional comparada, comienza con los trabajos de Burton Clark en la década de 1980 (Clark, 1986, 1987), solo desde la encuesta Carnegie sobre la profesión académica —Carnegie Survey of the Academic Profession—, aplicada entre 1991 y 1993 (Boyer, Altbach $\&$ Whitelaw, 1994; Schiefelbein, 1996), dicho estudio ha podido hacerse a partir de la administración de encuestas internacionales. Esta primera encuesta abarcó a 14 países, Chile entre ellos. Liderada por Ernst L. Boyer, permitió indagar sobre los factores externos que afectaban a las instituciones y, en consecuencia, a sus académicos a comienzos de la década de los 90, en un contexto de significativa expansión de la matrícula de la educación superior en todo el mundo, lo que, a su vez, generaba una mayor presión sobre el rol que debían cumplir los académicos enfrentados ahora a una población estudiantil no solo más numerosa sino también más diversa.

Una segunda encuesta de este tipo se aplicó en 2007, con 19 países colaborando en la investigación, entre los cuales no estuvo Chile. Esta vez la encuesta se denominó Changing Academic Profession (CAP), y puso foco en la relevancia del trabajo académico en un contexto de internacionalización y globalización, y de un marcado incremento de las regulaciones para el aseguramiento de la calidad (Höhle \& Teichler, 2012; Teichler et al., 2013).

La tercera encuesta (2018-2019) ha sido denominada Academic Profession in the Knowledge-Based Society (APIKS). El origen de la encuesta APIKS es un trabajo colaborativo que se inicia en 2014, en Helsinki, y que se desarrolla a través de una serie de workshops y encuentros anuales entre los equipos nacionales que tomaron a su cargo diseñarla e implementarla. El equipo chileno participó en tres de estos encuentros. Finalmente, durante 2018 y 2019 se aplicó la encuesta en 23 países, incluidos Alemania, Argentina, Finlandia, Croacia, China, Chile, EE.UU., Turquía Portugal, Reino Unido, México, Rusia, Taiwán, entre otros. El análisis de sus resultados continuará con una serie de talleres, inaugurados con una reunión de los equipos nacionales en Hiroshima, en 2019. 
La encuesta APIKS está compuesta por ocho módulos que buscan, por una parte, caracterizar la trayectoria y situación laboral de los académicos. Abarca también su desempeño en docencia, investigación y vinculación con el medio. Adicionalmente, cubre aspectos de gobierno y gestión académica. Especial atención se dirige a las etapas formativas en la profesión, a través de un módulo específico para dicho segmento. Por último, describe sus perfiles según variables demográficas, con perspectiva de género.

APIKS incluye preguntas de las versiones anteriores de la encuesta, para comparabilidad internacional e histórica. A su vez, cada país ha hecho sus adaptaciones nacionales de la encuesta común, que van desde la traducción del instrumento del inglés al idioma local, la adaptación de las preguntas a la estructura académica de cada país, hasta la incorporación de preguntas adicionales consideradas de interés para los respectivos países.

El presente artículo tiene por objeto presentar a la comunidad algunos de los resultados de la encuesta APIKS Chile, aplicada en 11 universidades entre fines de 2018 y comienzos de 2019. En esta primera publicación de resultados nos centraremos en describir el panorama que emerge en cuanto a las actividades de docencia, investigación y vinculación con el medio. Adicionalmente, presentaremos los principales hallazgos respecto de los académicos en etapas tempranas de su carrera. Por razones de espacio, los resultados que aquí se comunican corresponden a solo una parte de los que la encuesta entregó. Esperamos en futuras entregas completar el reporte de APIKS-Chile.

Las preguntas que estructuran el presente trabajo son las siguientes:

- ¿Cómo se distribuye el tiempo de trabajo entre las diversas actividades de los académicos?

- ¿Cómo se expresa la labor de investigación de los académicos?

- ¿Cómo se configura la labor de vinculación con el medio de los académicos? 
- ¿Qué tipo de apoyos institucionales existen para el desarrollo de académicos en las etapas tempranas de sus carreras?

El presente artículo comienza definiendo el marco conceptual desde el cual la literatura aborda la profesión académica. Luego se describen los principales aspectos metodológicos y de aplicación de la encuesta APIKS en Chile. A continuación se describen los primeros hallazgos encontrados, según las preguntas de investigación que guían este estudio. Finalmente, se presenta una discusión sobre la base de estos primeros resultados y la literatura sobre la carrera académica, proponiendo materias que pueden dar lugar a nuevas preguntas de investigación.

\section{Profesión académica: algunos elementos conceptuales}

La literatura sobre la profesión académica es vasta. Solo la serie de libros The Changing Academy - Changing Academic Profession in International Comparative Perspective, de la editorial Springer, ha publicado 20 volúmenes desde 2011. No pretendemos aquí hacer una revisión de toda esa literatura, sino solo de algunos aspectos de ella que podrían resultar más atingentes a los aspectos de la encuesta APIKS Chile que presentaremos más adelante. Para estos efectos, pondremos foco en tres áreas relacionadas con las preguntas de investigación: a) la primacía de la función de investigación sobre otras labores académicas y las condiciones en que se espera que ella se desenvuelva, b) la vinculación con el medio o "tercera misión" de la educación superior, y c) el desarrollo académico como responsabilidad institucional.

\subsection{Primacía de la investigación y condiciones para su desarrollo}

Aunque la noción moderna de la universidad se define por la asociación indisoluble entre docencia e investigación, que se expresa en el perfil y a labor de sus profesores, la producción de conocimiento se ha transformado en la labor central de la profesión académica contemporánea, tanto en el ámbito internacional, comenzando 
con el trabajo seminal de Jencks \& Riesman (1968), como en Chile (Bernasconi, 2007, 2008, 2012). Esta producción es la que se expresa en la publicación de artículos científicos en revistas indexadas (Teodorescu, 2000). Revistas con mayor reconocimiento internacional y menores tasas de aceptación de artículos se instalan como las más codiciadas por los investigadores. Algunos autores denominan a este fenómeno de creciente competitividad como "economía del prestigio" (Blackmore \& Kandiko, 2011; Kwiek, 2020). Este enfoque se potencia con una creciente cuantificación, estandarización, registros y competencias mediante rankings nacionales e internacionales (Ordorika \& Lloyd, 2015). Así, las instancias gubernamentales e institucionales generan políticas, iniciativas e incentivos para aumentar capacidades de producción científica (Altbach \& Salmi, 2011). Para los académicos y académicas esto se entiende como una priorización en tiempo y recursos hacia la investigación, y sus resultados como esenciales para los procesos de contratación y promoción en la carrera académica (Cowan \& Rossello, 2018; Rosinger, Taylor, Coco \& Slaughter, 2016).

La evidencia internacional muestra que la colaboración en investigación es uno de los mecanismos para aumentar la productividad (Ibarra, Kilduff \& Tsai, 2005; Lee \& Bozeman, 2005). Ya sea por el abordaje de problemas más complejos, la necesidad de cubrir un creciente estado del arte o sencillamente aumentar artificialmente la producción individual, el número de coautorías ha crecido en todas las disciplinas (Fanelli \& Larivière, 2016; Porter \& Rafols, 2009). En especial en países en la periferia de la producción científica internacional, como Chile (Guzmán-Valenzuela, 2019), la colaboración con investigadores de otras naciones se ha vuelto particularmente importante y objeto de políticas nacionales e internacionales (Canto \& Hannah, 2001; Cantwell, 2011; Celis \& Kim, 2018). Además, se ha ido instalando una necesidad de creación de conocimiento interdisciplinario, o de modo II, para obtener mayor pertinencia de las investigaciones (Gibbons, 2000; Wall \& Shankar, 2008). Esto también genera una necesidad de movilizar a los académicos para que colaboren con otras disciplinas (Celis \& Veliz, 2020). 
En países como Chile, a la necesidad de colaborar con pares nacionales e internacionales y de hacerlo de forma más interdisciplinaria, se suma la labor de instalar mayores capacidades locales de creación de conocimiento. Entre ellas, la de fundar y desarrollar programas de doctorado nacionales, que sean competitivos internacionalmente y pertinentes en el ámbito local, resulta crítica para el progreso científico de la nación (Celis \& Veliz, 2020; MuñozGarcia \& Bernasconi, 2020). Así, colaborar en la formación de futuros investigadores e investigadoras es también una función fundamental del trabajo académico.

Por último, es necesario señalar que la visión de reducir la producción de conocimiento al formato de publicaciones en revistas indexadas está bajo creciente cuestionamiento. Otras formas de evaluar y contabilizar la producción están siendo consideradas. Por ejemplo, la participación en patentes o licencias, como marcadores de hacer circular el conocimiento en los mercados o la sociedad en general.

\subsection{Vinculación con el medio}

Más allá de las funciones tradicionales de docencia e investigación, en las recientes décadas las instituciones de educación superior han puesto también el foco en innovar en la manera en la que llevan el conocimiento a la sociedad. En particular, los vínculos que existen entre las instituciones de educación superior y entidades externas, como la industria, el gobierno y la misma sociedad, han sido estudiados desde variadas perspectivas (Perkmann et al., 2013). Entre ellas se puede rescatar el concepto de "sistemas de innovación", que se define como: "funciones e interacciones complejas entre organizaciones e instituciones", en las que el gobierno, las empresas, universidades e institutos de investigación son consideradas organizaciones, mientras que las políticas y normas sociales serían instituciones (Cai, 2017).

Un precursor de esta apertura de la universidad hacia su medio es el conocido "modelo de triple hélice", que promueve una manera de comprender las interacciones dinámicas entre tres actores: 
universidad, industria y gobierno (Etzkowitz \& Leydesdorff, 1995), y se expresa, por ejemplo, en cómo el desarrollo de laboratorios de investigación en la industria origina la necesidad de académicos entrenados en métodos de experimentación.

La idea de "innovación" en educación superior se encuentra frecuentemente asociada en la literatura a la tercera misión, en la que lo central es la integración de lo social y lo comercial con la docencia y la investigación (Cai, 2017). Asimismo, otros autores expanden la idea de "tercera misión" más allá de la transferencia tecnológica, incluyendo en ella el desarrollo profesional constante de los académicos y la responsabilidad social de estos (Gaisch, Noemeyer \& Aichinger, 2019).

En Chile, la tercera misión o vinculación con el medio recoge estos mismos aspectos, en el sentido de que la interacción no es unidireccional sino multidireccional, lo cual demanda una coordinación integrada de diversas áreas y actividades que las instituciones de educación superior desarrollan. Sin embargo, existe un desarrollo heterogéneo de esta área en comparación con la docencia e investigación, siendo en la práctica un área considerada de menor relevancia, sin criterios claros y con una valoración institucional menor (Adán, Poblete, Angulo, Loncomilla y Muñoz, 2016). Más aún, las actividades externas que realizan los académicos no son necesariamente apreciadas de manera estandarizada y sistemática, a pesar de que muchas veces son consideradas para efecto de promoción académica (Adán et al., 2016).

Cabe señalar que la noción de "servicio a la comunidad externa" en Chile fue desarrollada por la Comisión Nacional de Acreditación, influenciada por el sistema europeo y norteamericano. En 2015, 60\% de las instituciones de educación superior en Chile tenían acreditada el área de vinculación con el medio.

\subsection{Desarrollo académico}

El desarrollo académico (DA), es decir, los procesos que apoyan el desarrollo de una carrera académica exitosa, ha evolucionado tanto en su forma como en el foco durante las últimas cinco décadas 
(Austin \& Sorcinelli, 2013). En un principio, las unidades de DA se enfocaban en temas puntuales, tales como gestionar los años sabáticos de los académicos. Hoy en día estas unidades ofrecen una cantidad de programas para apoyar el desarrollo de carrera académica $y$, en muchos países, se cuenta con profesionales especializados en estos temas. Como afirman Austin y Sorcinelli (2013), el desarrollo académico es una palanca clave para asegurar la calidad institucional y para apoyar los cambios que ocurren constantemente en la educación superior.

En este sentido, la literatura internacional nos entrega lineamientos para el DA en las instituciones de educación superior (Gappa, Austin \& Trice, 2007). En primer lugar, las restricciones presupuestarias y mayor rendición de cuentas (como el aseguramiento de la calidad) toman cada vez más protagonismo. Segundo, la diversidad de estudiantes a los que los académicos y las instituciones de educación se enfrentan, por ejemplo, estudiantes de primera generación, estudiantes internacionales, estudiantes con distintas orientaciones sexuales, etc. Adicionalmente, hoy son muy relevantes las oportunidades y desafíos del uso de la tecnología. Cómo las instituciones se adaptan a este contexto es de gran importancia. Del mismo modo, la interdisciplinariedad toma cada vez más protagonismo para resolver temas complejos. Por último, no podemos dejar pasar el cambio de las características de nuestros académicos y los cambios en los tipos de contratos.

En términos individuales, se ha estudiado cuáles son los tipos de roles y responsabilidades a que los académicos se ven enfrentados. Claramente, dependiendo del momento en el cual se está en la carrera académica, se plantean distintos desafíos. En la carrera temprana, marcada por el desafío de pasar a la categoría de profesor asociado, los académicos generalmente se sienten sobrepasados, lidiando por balancear las múltiples responsabilidades que la vida académica conlleva, como es la docencia, la investigación y la vinculación con el medio (Austin, 2002; Rice, Sorcinelli \& Austin, 2000). En esos momentos de la carrera se les puede apoyar mediante estrategias que promuevan las relaciones colegiadas en los departamentos. También las redes de apoyo son un punto crucial para el desarrollo profesional 
(O’Meara et al., 2008). Asimismo, los programas de mentorías son muy efectivos, así como las estrategias para balancear el trabajo y la vida personal (Austin, 2010). En etapas más avanzados de la carrera se puede ofrecer programas para facilitar la promoción a profesor titular, o bien que apunten a planificar la jubilación y la vida después de ella (Sorcinelli et al., 2006). En relación con los profesores-hora, Lyons (2007) y Tarr (2010) ofrecen buenas prácticas para apoyarlos, tales como programas para planificar el desarrollo profesional, apoyo en la docencia, recursos en línea y reconocimiento y premios por excelencia docente.

Es importante destacar que el DA también debiese apoyar a los académicos a desarrollar estrategias sobre el manejo del tiempo, priorización de responsabilidades y adecuado manejo del ambiente laboral (Austin \& Sorcinelli, 2013). Muchas unidades de DA ya proveen de programas, cuyo propósito es apoyar a los académicos en técnicas de docencia centradas en el estudiante y su aprendizaje activo (Sorcinelli et al., 2006), o incorporan el desarrollo de técnicas de liderazgo en sus ofertas de programas.

Con todo, no existe un único modelo apropiado de DA aplicable a todas las instituciones. Por el contrario, los académicos, administradores y directores de desarrollo académico deben considerar las especificidades de sus contextos institucionales. El desarrollo académico constituye un engranaje crucial para la calidad y para responder a las demandas complejas que hoy enfrentan las instituciones de educación superior (Austin \& Sorcinelli, 2013).

\section{Metodología: encuesta de la profesión académica APIKS Chile}

El desarrollo y aplicación de la encuesta APIKS Chile estuvo a cargo de un equipo de expertos conformado por académicos de la Universidad de Chile y la Pontificia Universidad Católica de Chile, constituido en 2014 para llevar a cabo este proyecto comparativo. Tomando como base la encuesta desarrollada por APIKS, se procedió a traducirla al español en coordinación con los equipos de Argentina y México, procurando ajustar el instrumento original, en inglés, al contexto 
de la carrera académica en América Latina y cuidando de mantener la mayor fidelidad posible respecto del cuestionario original, con el propósito de permitir la comparabilidad de los resultados entre los países.

La encuesta APIKS está compuesta de ocho dimensiones, y consta de 65 preguntas. Entre las temáticas abordadas se encuentra la percepción de los académicos respecto de sus motivaciones y preferencias por la docencia e investigación, su rol en el gobierno universitario, sus aportes a la sociedad y la relevancia de sus actividades de investigación, su compromiso con la academia, la conciliación de actividades domésticas con el trabajo según género, situación general del empleo, entre otras.

Con relación a la selección de la muestra, en 2017 se decidió considerar solo al sector universitario, dejando de lado el sector técnico-profesional de centros de formación técnica e institutos profesionales. Esta decisión se basa en que APIKS solo se aplica a académicos con al menos 23 horas de contrato, considera a la investigación como parte de las funciones del profesorado y asume que los que responden la encuesta tienen el grado de doctor, elementos todos que escapan al perfil de los docentes del sector técnico-profesional.

A principios de 2018 se pilotea la encuesta a través de un pequeño grupo de académicos $y$, posteriormente, se conforma una selección de 16 universidades chilenas representativa de la diversidad institucional del sector, incluyendo universidades estatales (CUECh), privadas en el Consejo de Rectores CRUCh (también conocidas como G9) y universidades privadas que participan del Sistema Único de Admisión (SUA) 6 . Se optó por considerar solo a las universidades privadas en el SUA debido a su mayor nivel

6 En Chile existen universidades estatales y universidades privadas. Todas las estatales, que se agrupan en el Consorcio de Universidades del Estado de Chile (CUECh) forman parte del Consejo de Rectores de las Universidades Chilenas (CRUCh). Entre las privadas, hay nueve de ellas (G9), las más antiguas, que también forman parte del CRUCh, y reciben subsidio (financiamiento) del Estado. Las otras universidades privadas, a la fecha de aplicación de APIKS, no forman parte del CRUCh, pero pueden participar en el sistema de ingreso a la universidad, Sistema Único de Admisión (SUA), que el CRUCh administra. 
de consolidación académica, toda vez que la encuesta asume la existencia en las universidades de cuerpos académicos de jornada completa estables y consolidados. Un elemento de conveniencia determinó la selección de esas 16 universidades: la cercanía del equipo APIKS Chile a los rectores de dichas instituciones, lo que favorecía la decisión de las universidades de ingresar al estudio. La invitación se dirigió a los rectores y vicerrectores académicos de esas universidades, y se operacionalizó designando como contrapartes técnicas a las direcciones de análisis institucional de cada una de ellas. Adicionalmente, se obtuvo la autorización ética de la Universidad de Chile (Facultad de Ciencias Físicas y Matemáticas), con el fin de dar garantía del buen uso y protección de la confidencialidad y anonimidad de la información que sería recolectada. Una vez recibida la aprobación, se procedió a enviar la encuesta a las contrapartes institucionales, quienes, a su vez, enviaron la encuesta al cuerpo académico contratado con 23 horas y más en sus universidades.

De las 16 universidades invitadas a participar en la encuesta en 2018, once ${ }^{7}$ accedieron a participar. Dada la gran heterogeneidad del sector universitario, se cauteló que hubiera al menos dos universidades en cada categoría, a lo que se sumó el tamaño de la universidad (número de alumnos) como criterio adicional. La aplicación de la encuesta se realizó entre el segundo semestre de 2018 y el primer semestre de 2019. Se aplicó online y se envió una invitación a responder la encuesta a más de 8.000 académicos de planta ordinaria o regular, con al menos 23 horas de contrato.

Se recibieron 1.850 respuestas en total. De ellas, 1.258 están completas. Los datos que aquí se informan corresponden a las respuestas completas. Mediante un análisis estadístico descriptivo, la encuesta fue analizada para cada dimensión de manera independiente. El detalle de las respuestas, según el tipo de institución a la que pertenecen los académicos, se presenta en la tabla 1.

7 Las once universidades participantes son: Universidad de Chile, Pontificia Universidad Católica de Chile, Universidad Austral de Chile, Pontificia Universidad Católica de Valparaíso, Universidad de Talca, Universidad Autónoma de Chile, Universidad del Desarrollo, Universidad San Sebastián, Universidad de los Andes, Universidad Tecnológica Metropolitana y Universidad Finis Terrae. 
Tabla 1:

Número de respuestas según tipo de Universidad (2018-2019)

\begin{tabular}{lccc}
\hline Tipo universidad & $\begin{array}{c}\mathrm{N}^{\circ} \text { Universidades } \\
\text { participantes }\end{array}$ & $\begin{array}{c}\mathrm{N}^{\circ} \text { Respuestas } \\
\text { totales }\end{array}$ & $\begin{array}{c}\mathrm{N}^{\circ} \text { Respuestas } \\
\text { completas }\end{array}$ \\
\hline TOTAL & 11 & 1.850 & 1.258 \\
$\begin{array}{l}\text { Universidades públicas } \\
\text { (CUECh) }\end{array}$ & 3 & 704 & 464 \\
Universidades privadas con & 3 & $(38 \%)$ & $(37 \%)$ \\
subsidio estatal (CRUCh) & & 334 & 252 \\
$\begin{array}{l}\text { Universidades privadas } \\
\text { (SUA) }\end{array}$ & 5 & $(18 \%)$ & $(20 \%)$ \\
& & 812 & 542 \\
& & $(44 \%)$ & $(43 \%)$ \\
\hline
\end{tabular}

Del total de académicos que respondieron la encuesta, 41,3\% corresponde a la categoría de profesor asistente, seguido de $27,4 \%$ de profesores asociados. El tercer grupo, correspondiente a los profesores titulares, representa 14,3\%, mientras que la categoría de profesor instructor alcanza 17\% del total. En cuanto a la distribución por sexo, del total de participantes $46 \%$ corresponde a mujeres y $54 \%$ a varones. Con relación a la edad, las mujeres, en promedio, son ligeramente menores que los hombres, siendo el promedio de edad de las académicas de 46,5 años y el de los académicos 48,9 años.

\section{Resultados seleccionados}

\subsection{Funciones que desempeñan los académicos}

En relación con las funciones que desempeñan los académicos, se les pidió indicar las horas semanales que dedican a las siguientes tareas: docencia, guía de tesis, investigación, gestión y vinculación con el medio. Casi un tercio (32\%) del tiempo corresponde al desempeño de labores de docencia. En segundo lugar aparecen las labores relacionadas con la gestión (26\% del tiempo). Le siguen las actividades de investigación, con 24\% de tiempo de dedicación. Más abajo se encuentran las relativas a la vinculación con el medio y guía de tesis, cada una con un 9\% de dedicación.

Dentro del ámbito de la docencia, se preguntó por el nivel en que ella se realiza: pregrado, posgrado o educación continua. El conjunto de académicos participantes en la encuesta APIKS en 
Chile dedica 58,2\% de su jornada de docencia al pregrado, 34,4\% a posgrado (magíster y doctorado), mientras que la educación continua presenta una menor proporción de dedicación docente, alcanzando un $13,4 \%$ del tiempo de los académicos (ver tabla 2 por tipo de universidades).

Tabla 2:

Porcentaje de tiempo de dedicación según tipo de docencia

\begin{tabular}{lcccc}
\hline Tipo de universidad & $\begin{array}{c}\text { Docencia } \\
\text { pregrado }\end{array}$ & $\begin{array}{c}\text { Docencia } \\
\text { magíster }\end{array}$ & $\begin{array}{c}\text { Docencia } \\
\text { doctorado }\end{array}$ & $\begin{array}{c}\text { Docencia } \\
\text { educación } \\
\text { continua }\end{array}$ \\
\hline $\begin{array}{l}\text { Universidades públicas (CUECh) } \\
\begin{array}{l}\text { Universidades privadas con } \\
\text { subsidio estatal (CRUCh) }\end{array}\end{array} \quad 56,4 \%$ & $19 \%$ & $18 \%$ & $14,3 \%$ \\
Universidades privadas (SUA) & $61,1 \%$ & $19 \%$ & $20,8 \%$ & $10,9 \%$ \\
\hline
\end{tabular}

Dependiendo del tipo de institución, la dedicación a la docencia en sus distintos niveles tiende a variar. No obstante lo anterior, se observa una distribución más o menos similar entre las universidades estatales (CUECh), las privadas del CRUCh y las privadas SUA, en tanto todas ellas se dedican mayoritariamente a la docencia de pregrado. La dedicación a la docencia de posgrado es menor en el caso de las universidades privadas SUA, siendo la docencia de doctorado casi la mitad de lo que dedican los académicos de las universidades públicas y privadas del Consejo de Rectores.

En cuanto a producción científica, los resultados muestran que las publicaciones en forma de artículos concentran los tipos de producción. La tabla 3 (o D $3^{8}$ ) muestra el promedio de la productividad científica y de creación en los últimos tres años, declarada por los participantes.

8 La numeración de las tablas y figuras con letra y número corresponde a las preguntas del cuestionario, y se indica aquí a efectos de comparabilidad con la misma pregunta en las encuestas de otros países. 
60 LA PROFESIÓN ACADÉMICA EN CHILE: PERSPECTIVAS DESDE UNA ENCUESTA INTERNACIONAL - A. Bernasconi, P. Berríos Cortés, P. Guzmán, D. Véliz, S. Celis

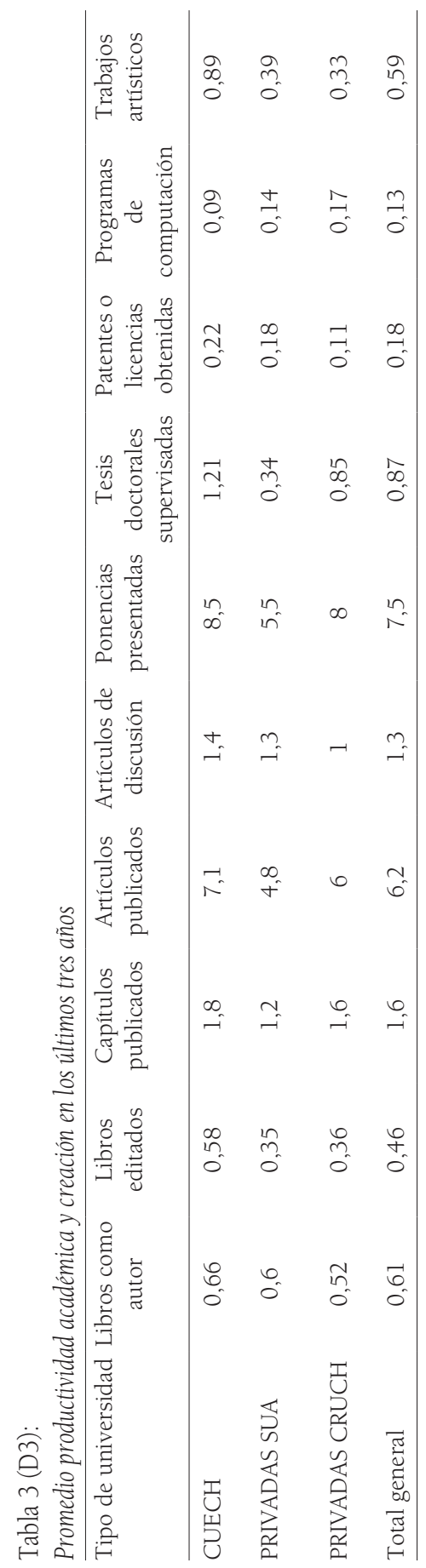


Las ponencias presentadas en congresos y los artículos publicados son las principales formas de producción en los tres tipos de instituciones estudiadas. Los académicos de universidades estatales (CUECh) declaran el mayor número de artículos (7,1 en tres años), seguidos por los de las privadas CRUCh, con 6 , y aquellos de las privadas SUA, con 4,8. Es interesante, aunque no sorpresivo, el patrón de correspondencia entre las ponencias en congresos y las publicaciones. Una suposición razonable es que las ponencias anteceden a las publicaciones. En muchas disciplinas, los trabajos de investigación en progreso se comparten y discuten con los pares en congresos, y luego se mejoran para llegar finalmente a la publicación. Este patrón sugiere que es importante apoyar la participación de los académicos para que asistan a congresos y compartan los trabajos con pares nacionales e internacionales. La tabla 3 (D3) también muestra la marcada diferencia con otras formas de producción. Muy por detrás de los artículos publicados, aparecen los capítulos de libro como el tercer producto más frecuente, con un promedio entre 1 y 2 (en tres años) para todas las instituciones. Sorprende el bajo número de tesis de doctorado guiadas, que va desde 0,3 en las privadas SUA a 1,2 en las instituciones estatales (CUECh). Estos números reflejan el aún incipiente desarrollo de los programas de doctorado en Chile. En último lugar aparecen patentes y licencias, programas computacionales y trabajos artísticos.

El panel de la figura 1 (D1) es elocuente en mostrar, como lo sugiere la literatura, cómo la colaboración académica es efectivamente un mecanismo esencial para el trabajo académico en Chile. Los porcentajes de colaboración son muy similares entre las universidades estatales (CUECh) y las privadas CRUCh. En todas las dimensiones de colaboración, las universidades privadas del SUA se encuentran más atrás que el resto. En las estatales (CUECh) y privadas CRUCh, sobre $85 \%$ de los académicos declara tener colaboración con pares en proyectos de investigación, principalmente de la misma institución. Sin embargo, se muestra que en Chile existe un gran porcentaje de colaboración entre académicos de distintas instituciones, desde $66,1 \%$ de las privadas SUA hasta $75,5 \%$ en las privadas CRUCh. Otro porcentaje relevante es la colaboración con pares internacionales, la 
que va desde $55,8 \%$ hasta $78 \%$ en las privadas SUA y CRUCh, respectivamente.
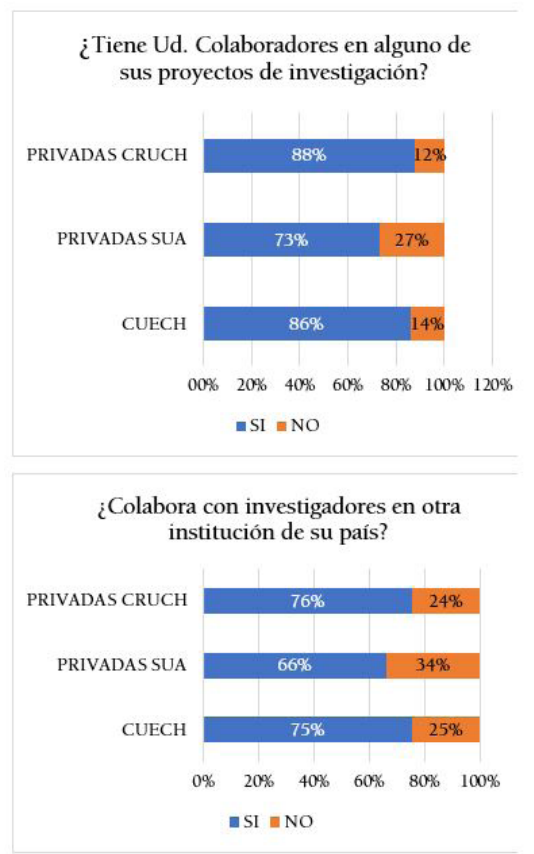

Figura 1 (D1):

Colaboración interna y externa en investigación.
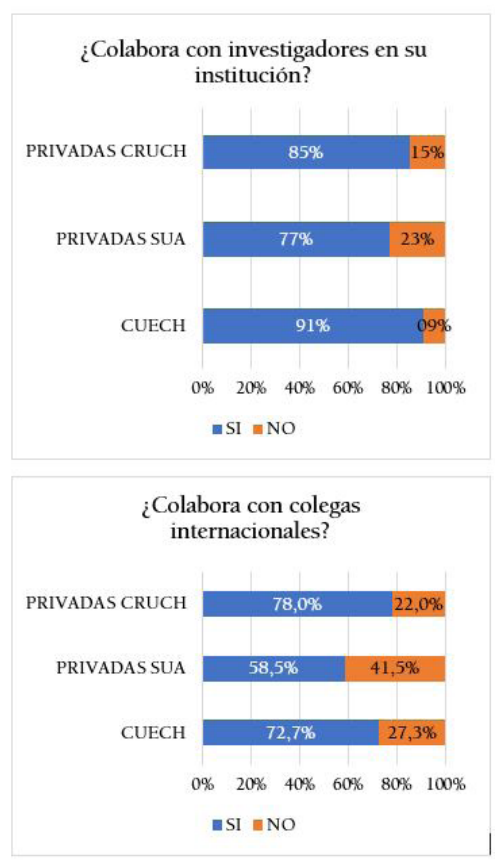

La colaboración entre académicos de distintas disciplinas también muestra altos porcentajes, desde $66,9 \%$ en las privadas SUA hasta 80,4\% en las estatales (CUECh).

Por último, y reflejando la figura 2 (D2) el mismo fenómeno observado en la tabla 3 (D3), menos de la mitad de los académicos informa colaboración con estudiantes de doctorado. 


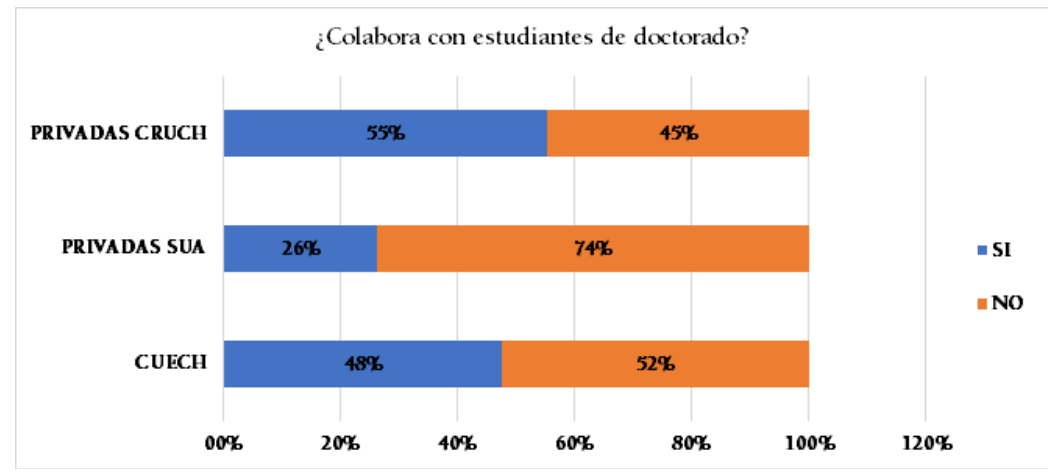

Figura 2 (D2):

Colaboración con estudiantes de doctorado.

En el ámbito de la vinculación con el medio, la figura 3 (E2) muestra que los académicos pertenecientes a las tres clasificaciones de universidades generan principalmente alianzas con otras instituciones de educación superior. El tipo de socio que ocupa el segundo lugar en frecuencia, tanto para las universidades privadas CRUCh como para las estatales (CUECh), es el gobierno. En cambio, para las privadas SUA el segundo lugar en alianzas se da con organizaciones sin fines de lucro. Este último tipo de organizaciones resulta ser el tercer socio para las privadas CRUCh y estatales (CUECh), mientras que para las universidades privadas SUA el tercer socio es el gobierno.

De manera medianamente frecuente se encuentra como alianza a la empresa. Esto es, en cuarto lugar para las universidades privadas CRUCh y privadas SUA, y en quinto lugar para las universidades estatales (CUECh). Asimismo, otros socios que resultan menos habituales para todas las universidades participantes son las instituciones públicas de investigación. Los académicos de los tres tipos de universidades declaran formar, en último lugar, vínculos con instituciones privadas de investigación ${ }^{9}$.

9 Las instituciones públicas y privadas de investigación son entidades no universitarias que realizan funciones de investigación, tales como laboratorios, institutos CORFO, hospitales, fundaciones o think tanks. 


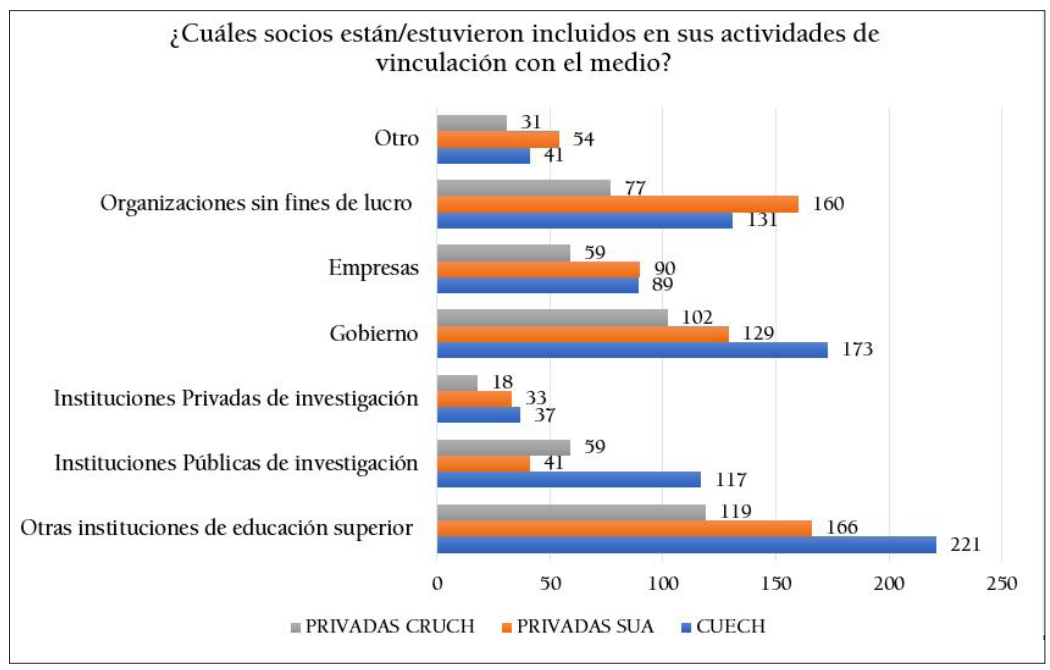

Figura 3 (E2):

Tipo de socios en actividades de vinculación con el medio (número de vinculaciones).

En suma, los resultados revelan que los académicos de todas las instituciones encuestadas generan vínculos mayoritariamente con otras instituciones de educación superior, seguidas principalmente por organizaciones sin fines de lucro y el gobierno. Los vínculos menos frecuentes son con instituciones privadas de investigación.

En relación con la contribución que, a juicio de los académicos, hacen las actividades de vinculación con el medio, se observa en los tres tipos de universidades que el mayor aporte se dirige hacia la comunicad local/regional (estatales, 67\%; privadas SUA, 79,9\%; privadas CRUCh, $77,1 \%$ ) y hacia la sociedad nacional (estatales, 65,1\%; privadas SUA, 67,9\%; privadas CRUCh, 70,1\%). La contribución hacia la industria es la de menor frecuencia: solo 22,3\% de los académicos de las universidades estatales (CUECh) declara que su aporte sea bastante en esta área, 34,5\% declara lo mismo de entre las privadas SUA y 22,9\% entre las privadas CRUCh. La contribución a la sociedad nacional duplica a la contribución a la sociedad internacional, a juicio de los encuestados. 
En definitiva, existe homogeneidad entre los tres tipos de universidades en cuanto a la percepción que sus académicos tienen del aporte de sus labores de vinculación con el medio en la comunidad local, la industria, la sociedad nacional y la sociedad internacional. Predominan las contribuciones a la comunidad local y a la sociedad nacional, y se estiman menores las contribuciones a la empresa y a la sociedad internacional.

\subsection{Académicos en etapas formativas}

Para APIKS, los académicos en etapas formativas son los que se encuentran en categorías de instructor o asistente. Solo quienes se encontraban en estas categorías respondieron este módulo de la encuesta. Los resultados en materia de desarrollo académico muestran que las oportunidades de mentoría son aún incipientes en las universidades en Chile. Con todo, los hallazgos sugieren que este tipo de apoyos son más frecuentes en las universidades privadas del SUA $(27,9 \%$ de sus académicos declara que tiene mentorías disponibles cuando las necesitan), en comparación con 13,4\% en las estatales (CUECh) y 18,9\% en las universidades privadas del CRUCh. Entonces, tenemos que entre 70\% y $80 \%$ de los académicos reportaron que no están ni de acuerdo ni en desacuerdo, o están en desacuerdo, con la afirmación de que disponen de mentorías en su institución. La poca presencia de estos apoyos a la carrera académica en nuestro país contrasta con la evidencia internacional, que indica que ellos son importantes para el éxito profesional de los académicos.

No obstante, una pregunta más amplia recoge que 53,1\% de los académicos de las universidades privadas de SUA señala que se encuentra bien respaldado o acompañado en su carrera académica. Por el contrario, en las universidades del CUECh el porcentaje es menos de un tercio $(31,7 \%)$, casi idéntico al de las universidades privadas del CRUCh $(31,8 \%)$. Estos resultados pueden deberse a un más deliberado apoyo de las universidades privadas SUA a sus profesores o, también, a que siendo en ellas los profesores de alta dedicación un porcentaje minoritario del total de los académicos, aquellos se sienten en una posición de privilegio respecto de los profesores-hora si se están comparando con éstos. 
Por último, con relación a las oportunidades para generar redes de trabajo, se aprecia una tendencia positiva en los tres tipos de universidades respecto de contar con buenas ocasiones para tener contacto social y desarrollar redes en sus unidades académicas. Los académicos de las universidades privadas del SUA son los que muestran un mayor nivel de acuerdo en este punto, con 62,3\%; le siguen, con $53,4 \%$, los de las universidades privadas del CRUCh y con un menor nivel de acuerdo los de las universidades estatales (CUECh). Estas cifras sugieren que las universidades donde los académicos se encuentran más respaldados en su carrera académica también son las que generan buenas oportunidades de desarrollar redes en su unidad académica, algo fundamental para el desarrollo profesional (O’Meara et al., 2008).

\section{Discusión y conclusiones}

En sus tres décadas de vigencia, las encuestas sobre la profesión académica han generado resultados internacionales interesantes, destacando a la vez la importancia del contexto a la hora de analizar la profesión académica en cada país (Huang, 2019). Vistas en retrospectiva, las encuestas sobre la profesión académica se han convertido en un instrumento que aporta una perspectiva histórica global de la evolución de esta profesión. Cada aplicación ha tenido su sello epocal y ha sido influida por nociones sobre la profesión académica en crisis (Altbach \& Finkelstein, 1997), en proceso de cambio (Goastellec et al., 2011; Höhle \& Teichler, 2012) y, más recientemente, como una profesión inherente a la sociedad del conocimiento (UNESCO, 2005).

La encuesta APIKS Chile ha generado un enorme caudal de datos, los que, por razones de espacio, no pueden ser presentados en un solo artículo. En este primer informe hemos optado por compartir algunos de los resultados acerca de las labores que desempeñan los académicos, así como poner un foco en etapas tempranas de sus carreras.

En cuanto a las labores que desempeñan, no encontramos evidencia de que las labores de investigación hayan desplazado a la 
docencia como atención principal de los académicos. Lo que llama la atención es que una cuarta parte del tiempo se dedique a labores de gestión. Esto puede deberse a que la gestión de la investigación, en el sentido de administrar proyectos, haya sido sumada por los académicos al ítem "gestión" y no al de investigación (la pregunta no permite dilucidar esto). Alternativamente, la cifra podría reflejar una baja eficiencia de las universidades chilenas en liberar a los académicos de labores de gestión, las que en los países desarrollados se hacen descansar en cuadros profesionales especializados en administración de procesos.

Donde sí aparecen los académicos chilenos alineados con las tendencias internacionales es en su preferencia por los artículos de revistas científicas como modo de comunicar resultados de investigación. Es clara la postergación de los libros y los capítulos de libros, todo ello en consonancia con la estructura de recompensas que han diseñado las propias instituciones, así como los modos de evaluar productividad en la Agencia Nacional de Investigación (ex CONICYT). También, en línea con la literatura, aparece la penetración de las colaboraciones nacionales e internacionales como modo de producción de conocimiento, incluyendo a colegas de otras disciplinas.

Como grupo, las universidades privadas SUA presentes en la encuesta muestran, en general, una menor productividad científica y menor grado de colaboración en investigación que lo que informan los académicos de las universidades privadas CRUCh y en las estatales. Esto es consistente con la más reciente instalación de capacidades de investigación en aquellas que en estas. Estos resultados, además, son consistentes con la distribución de proyectos de investigación y de publicaciones a nivel nacional entre los tres tipos de instituciones.

Por otro lado, es interesante el espacio que la vinculación con el medio está ocupando en la agenda de los académicos (casi 10\% de la jornada), fenómeno que puede estar relacionado con la inclusión de la vinculación como área de acreditación institucional opcional desde 2006. Debe repararse, eso sí, en que hay un traslape entre labores de vinculación con el medio y la docencia en educación continua 
informada en la tabla 2. En otras palabras, es posible que mucho del tiempo que se declara dedicado a vinculación con el medio sea, en realidad, docencia de extensión. En todo caso, ésta suele considerarse por las universidades como acciones de vinculación con el medio, de modo que la encuesta recoge este doble rol de la docencia de extensión.

La información que entrega la encuesta sobre las alianzas con el medio externo es llamativa: si se excluye a las otras instituciones de educación superior, el principal socio es el gobierno, luego las ONG y fundaciones y, en tercer lugar, la empresa. Las universidades estatales tienen ventaja en la colaboración con el gobierno y las entidades públicas de investigación, en tanto las privadas del CRUCh lideran en colaboraciones con las ONG. Esto podría reflejar patrones institucionales de colaboración, más que solamente las preferencias individuales de los académicos.

Por último, los académicos en etapas formativas en las universidades estatales declaran tener menores apoyos al desarrollo de sus carreras académicas que los de las universidades privadas SUA y privadas CRUCh, en todas las dimensiones de la pregunta. La instalación de esquemas formales de mentoría, que han mostrado su efectividad en los países desarrollados, podría ser una respuesta a este problema.

\section{Referencias}

Adán, L., Poblete, F., Angulo, C., Loncomilla, L. y Muñoz, Z. (2016). Aseguramiento de la Calidad en Educación Superior. La función de Vinculación o Tercera Misión en el contexto de la Educación Superior Chilena. Cuadernos de Investigación $N^{\circ} 1$. Santiago de Chile: Comisión Nacional de Acreditación.

Altbach, P. G. (1991). The Academic Profession. In International Higher Education: An Encyclopedia (pp. 23-46). New York: Garland Publishers.

Altbach, Ph. G. \& Finkelstein, M. J. (1997). The Academic Profession - The Professoriate in Crisis. Abingdon: Routledge

Altbach, Ph. G. \& Lewis, L. S. (1996). The Academic Profession in International Perspective. In The International Academic Profession: 
Portraits of Fourteen Countries. Princeton: The Carnegie Foundation for the Advancement of Teaching.

Altbach, P. G., Salmi, J. (Eds.), 2011. The Road to Academic Excellence: The Making of World-class Research Universities. Washington, DC: The World Bank,

Austin, A. E. \& Sorcinelli, M. D. (2013). The future of faculty development: Where are we going? New directions for teaching and learning, (133), 85-97.

Austin, A. E. (2002). Preparing the Next Generation of Faculty: Graduate Education as Socialization to the Academic Career. The Journal of Higher Education, 73(2), 94-122.

Bedeian, A. G., Cavazos, D. E., Hunt, J. G., Jauch, L. R. (2010). Doctoral degree prestige. Academy of Management Learning \& Education, 9(1), 11-25.

Blackmore, P. \& Kandiko, C. B. (2011). Motivation in academic life: a prestige economy. Research in Post-Compulsory Education, 16(4), 399-411.

Bernasconi, A. (2012). Gestión del cuerpo académico en un contexto de mercado: el caso de Chile. En N. Fernández Lamarra y M. Marquina (comps.), El futuro de la profesión académica. Desafíos para los países emergentes (pp. 153-167). Buenos Aires: Editorial de la Universidad Nacional de Tres de Febrero.

Bernasconi, A. (Ed.). (2015). La educación superior de Chile: Transformación, desarrollo y crisis. Santiago de Chile: Ediciones UC.

Berrios, P. (2015). La profesion académica en chile: crecimiento y profesionalizacion. En A. Bernasconi (Ed.), La Educación Superior De Chile: Transformación, Desarrollo Y Crisis (pp. 345-370). Santiago de Chile: Ediciones UC.

Boyer, E. L., Altbach, P. G. \& Whitelaw, M. J. (1994). The Academic Profession. An International Perspective. A special Report. Princeton: The Carnegie Foundation for the Advancement of Teaching.

Cai, Y. (2017). From an Analytical Framework for Understanding the Innovation Process in Higher Education to an Emerging Research Field of Innovations in Higher Education. The Review of Higher Education, 40(4), 585-616.

Canto, I. \& Hannah, J. (2001). A Partnership of Equals? Academic Collaboration between the United Kingdom and Brazil. Journal of Studies in International Education, 5(1), 26-41. 
Cantwell, B. (2011). Transnational mobility and international academic employment: gatekeeping in an academic competition arena. Minerva, 49(4), 425-445.

Celis, S. \& Kim, J. (2018). International patterns of faculty hiring, faculty collaboration, and institutional prestige in engineering: A global perspective from Chile and Korea. Research Policy, 47(3), 573-582.

Celis, S. \& Veliz, D. (2020). A decade of Chilean graduate program accreditation: A push for internationalization and issues of multidisciplinarity. Higher Education Policy. DOI: https://doi. org/10.1057/s41307-020-00198-7

Clark, B. R. (1987). The academic life: Small worlds, different worlds. Princeton: The Carnegie Foundation for the Advancement of Teaching.

Clark, B. R. (1986). The higher education system: Academic organization in crossnational perspective. Berkeley: University of California Press.

Cowan, R. \& Rossello, G. (2018). Emergent structures in faculty hiring networks, and the effects of mobility on academic performance. Scientometrics, 117(1), 527-562.

Enders, J. \& Musselin, C. (2008). Back to the Future? The Academic Professions in the 21st Century. In Higher Education to 2030. Volume 1: Demography (pp. 125-150). OECD.

Etzkowitz, H. \& Leydesdorff, L. (1995). The Triple Helix —UniversityIndustry-Government relations: A laboratory for knowledge based economic development. EASST Review, 14(1), 14-19.

Fanelli, D. \& Larivière, V. (2016). Researchers' Individual Publication Rate Has Not Increased in a Century. PloS One, 11(3), e0149504.

Gappa, J. M., Austin A. E. \& Trice, A. G. (2007). Rethinking Faculty Work: Higher Education's Strategic Imperative. San Francisco: Jossey-Bass.

Gaisch, M., Noemeyer, D. \& Aichinger, R. (2019). Third mission activities at Austrian universities of applied sciences: Results from an expert survey. Publications, 7(57), 1-14. DOI: https://doi.org/10.3390/ publications 7030057

Gibbons, M. (2000). Mode 2 society and the emergence of context-sensitive science. Science \& Public Policy, 27(3), 159-163.

Gillespie, K. J., D. L. Robertson, and Associates. (Eds). (2010). A Guide to Faculty Development, 2nd ed. San Francisco: Jossey-Bass.

Goastellec, G., Park, E., Ates, G. \& Toffel, K. (2011). Academic Markets, Academic Careers: Where Do We Stand? In The Academic Profession in Europe: Responses to Societal Challenges. Dordrecht: Springer. 
Guzmán-Valenzuela, C. (2019). Values and the international collaborative research in higher education: negotiating epistemic power between the Global South and the Global North. In P. Gibbs, J. Jameson \& A. Elwick (editors), Values of, and in, the University in a Time of Uncertainty (pp. 137-153). Dordrecht: Springer.

Höhle, E. \& Teichler, U. (2012). The Academic Profession in the Light of Comparative Surveys. In The Changing Academy - The Changing Academic Profession in International Comparative Perspective (pp. 23-38). Springer. Recuperado de https://link.springer.com/chapt er/10.1007/978-94-007-4614-5_3

Huang, F. (2019, April). Changing attitudes to university teaching and research. University World News. Recuperado de https://www. universityworldnews.com/post.php?story=20190408143502971

Ibarra, H., Kilduff, M. \& Tsai, W. (2005). Zooming In and Out: Connecting Individuals and Collectivities at the Frontiers of Organizational Network Research. Organization Science, 16(4), 359-371.

Jencks, C. \& Riesman, D. 1968. The Academic Revolution. Garden City: Doubleday.

Kwiek, M. (2020). The prestige economy of higher education journals: a quantitative approach. Higher Education. DOI: https://doi.org/10.1007/ s10734-020-00553-y

Lee, S. \& Bozeman, B. (2005). The Impact of Research Collaboration on Scientific Productivity. Social Studies of Science, 35(5), 673-702.

Levine, A. 2010. Higher Education at a Crossroads. In Earl Pullias Lecture in Higher Education. Los Angeles: Center for Higher Education Policy Analysis, Rossier School of Education, University of Southern California.

Lyons, R. E. (Ed). (2007). Best Practices for Supporting Adjunct Faculty. Bolton, Mass.: Anker Press.

Muñoz-Garcia, A. L. \& Bernasconi, A. (2020). Reassessing the progress of doctoral education in Chile. In M. Yudkevich, P. Altbach \& H. de Wit. Trends and issues in doctoral education (pp. 414-441). Thousand Oaks: SAGE Studies in Higher Education.

Musselin, C. (2007). The Transformation of Academic Work: Facts and Analysis. Center for Studies in Higher Education, University of California, Berkeley.

O'Meara, K. A., Terosky, A. L. P. \& Neumann, A. (2008). Faculty careers and work lives: a professional growth perspective. ASHE Higher Education Report, 34(3). San Francisco, CA: Jossey-Bass. 
Ordorika, I. \& Lloyd, M. (2015). International rankings and the contest for university hegemony. Journal of Education Policy, 30(3), 385-405.

Perkmann, M., Tartari, V., McKelvey, M., Autio, E., Broström, A., D’Este, P., ... Sobrero, M. (2013). Academic engagement and commercialisation: A review of the literature on university-industry relations. Research Policy, 42(2), 423-442. DOI: https://doi.org/10.1016/j.respol.2012.09.007

Porter, A. L., \& Rafols, I. (2009). Is science becoming more interdisciplinary? Measuring and mapping six research fields over time. Scientometrics, 81(3), 719 .

Rice, R. E., Sorcinelli M. D. \& Austin, A. E. (2000). Heeding New Voices: Academic Careers for a New Generation. Washington, D.C.: American Association of Higher Education.

Rosinger, K. O., Taylor, B. J., Coco, L. \& Slaughter, S. (2016). Organizational Segmentation and the Prestige Economy: Deprofessionalization in High- and Low-Resource Departments. The Journal of Higher Education, 87(1), 27-54.

Schiefelbein, E. (1996). The Chilean Academic Profession: Six Policy Issues. In P.G. Altbach (Ed.), The International Academic Profession: Portraits of Fourteen Countries (pp. 281-306). The Carnegie Foundation for the Advancement of Teaching.

Sorcinelli, M. D., Austin, A. E., Eddy, P. \& Beach, A. 2006. Creating the Future of Faculty Development: Learning From the Past, Understanding the Present. Bolton, Mass.: Anker Press.

Tarr, T. A. (2010). Working with Adjunct Faculty Members. In K. J. Gillespie, D. L. Robertson, and associates, A Guide to Faculty Development, 2nd ed. (pp. 347-362). San Francisco: Jossey-Bass.

Teichler, U., Arimoto, A. \& Cummings, W. K. (2013). Changing academic profession: Major findings of a comparative survey. Dordrecht: Springer.

Teodorescu, D. (2000). Correlates of faculty publication productivity: A crossnational analysis. Higher Education, 39, 201-222.

UNESCO. (n.d.). UNESCO UIS Glossary. Recuperado de http://uis.unesco. org/en/glossary, accessed 11 March 2020.

UNESCO (2005). Towards knowledge societies: Paris: UNESCO Publishing..

Wall, S. \& Shankar, I. (2008). Adventures in transdisciplinary learning. Studies in Higher Education, 33(5), 551-565.

Recibido: 23/10/2020

Aceptado: 13/01/2021 